\title{
Relationship between Mechanical Absorption and Birefringence of Regenerated Cellulose Solid in Solvent
}

\author{
Rumiko FujIoka, ${ }^{*, * *}$ Chika IshiI, ${ }^{*}$ Ayako Mori, ${ }^{*}$ Sei-ichi Manabe, ${ }^{*}$ and Kiyohisa Imada** \\ * Faculty of Human Environmental Science, Fukuoka Women's University, \\ 1-1-I Kasumigaoka, Higashiku, Fukuoka 813-8529, Japan \\ ** Faculty of Engineering, Miyazaki University, \\ 1-1 Gakuenkibana-nisi Miyazaki 889-2155, Japan
}

(Received September 18, 1997)

\begin{abstract}
This study correlates the temperature dependence of birefringence $\Delta n$ of a regenerated cellulose solid in a solvent with thermal chain motion evaluated from mechanical loss tangent $\tan \delta$. Two regenerated cellulose hollow fibers composed of fine spherical particles (primary particles) from 10 to $20 \mathrm{~nm}$ in size were used. Solvents as immersing media were methanol, ethanol, $n$-propanol, and ethylene glycol. The temperature dependence of $\Delta n$ in the solvent was measured by the freezing method and the equilibrium method proposed by us. [Bull. Fac. Human Environ. Sci., Fukuoka Women's Univ., 28, 37 (1997)] (1) $\Delta n v s$. temperature curves by the freezing method showed a few peaks at the temperature region where $\tan \delta$ vs. temperature curve showed peaks or a shoulder. (2) Peak value varied with immersing media and freezing conditions. (3) For the sample having anisotropic orientation of the working direction of the intermolecular hydrogen bond, a few peaks were observed in the case of the freezing method at $\beta_{\mathrm{a}}$ absorption due to a local twisting motion of a main chain. (4) In the lower temperature region between $\beta_{\mathrm{a}}$ absorption and $\alpha_{\mathrm{a}}$ absorption, due to a micro-Brownian motion of a main chain, $\Delta n$ was reflected in molecular interactions between the medium and cellulose molecules. In the temperature region of $\alpha_{a}$ absorption, a few peaks of $\Delta n$ appears in both methods. $\Delta n$ peaks thus correlate closely with the onsets of mechanical absorption.

KEY WORDS Regenerated Cellulose / Solvent / Birefringence / Diffusion / Freezing Method / Equilibrium Method / Mechanical Absorption /
\end{abstract}

The higher order structure of a polymer solid influences significantly diffusion. The region into which a particle and a molecule diffuse should be discussed from two standpoints: (1) whether the geometrical size of the space is enough or not for the diffusion and (2) whether the micro-Brownian motion of a cellulose chain is activated sufficiently or not. This region is referred to as the diffusible region. It is important in studying the particle diffusion to know higher order structures of polymer solids and thermal molecular motion of the polymer chains.

The thermal molecular motion may be influenced by the particle diffused. In spite of the necessity of these investigations as for the diffusible region and the thermal molecular motion activated by the particle diffused, few investigations have been carried out so far. ${ }^{1}$ This is because there is no way for measurement of the effect of particles on motion and evaluation of the diffusible region.

We proposed in the previous paper ${ }^{2}$ a viscoelastic method for evaluation of the diffusible region and change in the thermal molecular motion of a polymer solid due to the diffusion of the particle. The diffusible region and solvent content in a given amorphous region are evaluated through the method. ${ }^{3}$

To evaluate thermal molecular motion of the chain in the medium containing a particle and the diffusional behavior of the particle, recently we proposed the method for the measurement of the temperature dependence of birefringence $\Delta n$ in a liquid medium. ${ }^{4,5}$ We proved the possibility of evaluating the thermal molecular motions and the diffusion of a solvent by the following three procedures: (1) freezing of thermal molecular motions (referred to as freezing method), (2) diffusional equilibrium (equilibrium method), (3) step-wise excita- tion (transitional method). Through these methods, we evaluate intermolecular interactions between particle and polymer chain.

The theoretical background of $\Delta n$ proposed so far is not enough for explanation of the temperature dependence of $\Delta n$ observed in a solvent. $\Delta n$ has been regarded as the sum ${ }^{6}$ of (a) $\Delta n$ due to the orientation of the molecular chains along a fiber axis in a crystalline and a non crystalline regions and (b)structural birefringence. Experimental $\Delta n$ taught us that the influence of the plane orientation and thermal molecular motions on $\Delta n$ should be added to the contributions of (a) and (b). ${ }^{7,8}$

This paper shows the temperature dependence of $\Delta n$ and dynamic viscoelasticities in a medium for two typical regenerated cellulose hollow fibers whose higher order structure has been well-investigated. We establish the relationship between the curves of $\Delta n v s$. temperature and thermal molecular motion for a regenerated cellulose solid in a medium containing a particle, and finally show the validity of the measurement of the curves of $\Delta n v s$. temperature in the medium containing the particle.

\section{EXPERIMENTAL}

\section{Sample Preparation}

Regenerated Cellulose Fiber. ${ }^{2,9}$ (1) Porous Cuprammonium Regenerated Cellulose Hollow Fiber (BMM40): BMM40 was prepared as reported previously. ${ }^{2}$ Mean pore size was $40 \mathrm{~nm} .{ }^{9}$ The higher order structure of BMM40 was represented by a stack structure of the secondary particle of $c a .75 \mathrm{~nm}$ in size. The secondary particle was composed of aggregates of primary particles of $c a .15 \mathrm{~nm}$ in size. BMM40 was almost non crystalline and showed little orientation of molecular chains along 
(1)

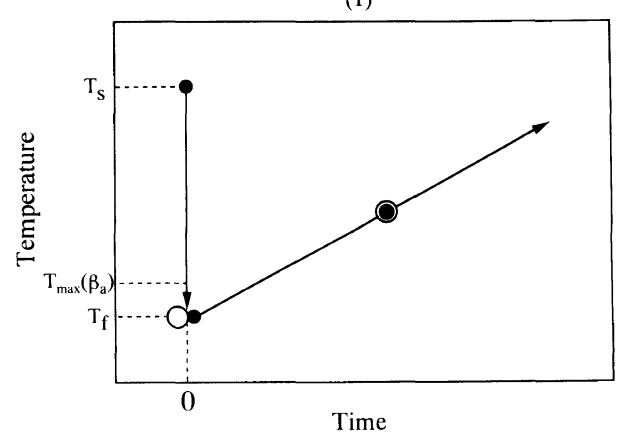

(2)

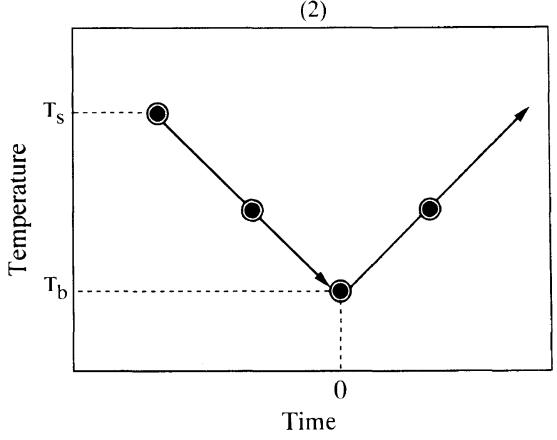

Figure 1. Schematic representation of change in temperature of a sample (polymer solid) and an immersing solvent with time: (1) Freezing method: Arrows indicate the direction of the temperature change. sample (polymer solid); $\bigcirc$, solvent; $\odot$, sample in an immersing solvent at equilibrium; $\bigcirc$, sample in the neighborhood of a solvent; $T_{\mathrm{s}}$, temperature of holding a sample and/or a solvent before measurement; $T_{\mathrm{f}}$, temperature of freezing of thermal molecular motion of a polymer chain; $T_{\mathrm{b}}$, bottom temperature of cooling a sample and/or solvent. $T_{\max }\left(\beta_{\mathrm{a}}\right)$, peak temperature of $\beta_{\mathrm{a}}$ absorption. (1) freezing method; (2) equilibrium method.

a fiber axis ${ }^{9}$ (this orientation is referred to as chain orientation). The crystal plane (110) in a crystalline region and/or the working direction of the intermolecular hydrogen bond in a non crystalline region were oriented in the circumferential direction of the hollow fiber in the case of BMM $40 .{ }^{10}$ Increase in the crystallinity with storage time for BMM40 was observed. ${ }^{11} \mathrm{We}$ distinguished BMM40 as BMM40(1) and BMM40(2). BMM40(2) indicated that about 180 days passed at $R H=0 \%$ and free of tension after BMM40(1) was used. (2)Virus Removal Hollow Fiber (BMM35): BMM35 was prepared by the similar procedure of BMM40 and was provided from Asahi Chemical Ind. Co., Ltd., Japan. Mean pore size was $35 \mathrm{~nm}$. The higher order structure of BMM35 was similar to BMM40. BMM35 had a higher degree of chain orientation than that of BMM40, and the plane orientation of BMM35 held at a slightly lower level than that of BMM 40 . These two samples were kept in a desicator packed with dry silicagel before use for more than a week.

Solvent as a Particle. The following solvents were used as immersing media during measurement of the $\Delta n$ and dynamic viscoelasticity: methanol, ethanol, $n$-propanol, ethylene glycol. These were guaranteed reagent grade manufactured by Wako Pure Chemical Ind. Ltd. (Japan).

\section{Measurements}

Temperature Dependence of $\Delta n$ in Solvent. $\Delta n$ vs. temperature curves were measured with heating/cool-
Table I. $T_{\mathrm{s}}$ and $T_{\mathrm{f}}$ used in the freezing method

\begin{tabular}{lccc}
\hline \multicolumn{1}{c}{ Solvent } & Sample & $T_{\mathrm{s}} / \mathrm{K}$ & $T_{\mathrm{f}} / \mathrm{K}$ \\
\hline \multirow{2}{*}{ Methanol } & BMM40 & 296 & 173 \\
& BMM35 & $296,493,543$ & 173 \\
\hline Ethanol & BMM40 & 296 & 163 \\
\hline$n$-Propanol & BMM40 & 296 & 163 \\
\hline
\end{tabular}

$T_{\mathrm{s}}$, temperature of holding a sample and/or a solvent before measurement; $T_{\mathrm{f}}$, temperature of freezing thermal molecular motion of a polymer chain.

Table II. $T_{\mathrm{s}}$ and $T_{\mathrm{b}}$ used in the equilibrium method

\begin{tabular}{lccc}
\multicolumn{1}{c}{ Solvent } & Sample & $T_{\mathrm{s}} / \mathrm{K}$ & $T_{\mathrm{b}} / \mathrm{K}$ \\
\hline Methanol & BMM40 & 296 & 173 \\
\hline Ethanol & BMM40 & 296 & 163 \\
\hline$n$-Propanol & BMM40 & 296 & 163 \\
\hline Ethylene Glycol & BMM35 & $253,296,373$ & 250 \\
\hline
\end{tabular}

$T_{\mathrm{s}}$, temperature of starting a sample and/or solvent; $T_{\mathrm{b}}$, bottom temperature of cooling a sample and/or solvent.

ing equipment ${ }^{4}$ installed with a polarized microscope. Two typical methods of the freezing method ${ }^{5}$ and the equilibrium method ${ }^{5}$ were used. The thermal histories employed in both methods are shown in Figure 1. $\Delta n$ was measured by Bereck's compensator. (1) Freezing method $^{5}$ : The sample was kept at the temperature $T_{\mathrm{s}}$ for $10 \mathrm{~min}$, and then was quenched into the solvent kept for $10 \mathrm{~min}$ at temperature $T_{\mathrm{f}}$ where most thermal molecular motion of polymer chains was frozen. Changes in $\Delta n$ were measured as a function of temperature under heating rate of $5 \mathrm{~K} \mathrm{~min}^{-1}$. All samples, and solvents, $T_{\mathrm{s}}$, and $T_{\mathrm{f}}$ are summarized in Table I. (2) Equilibrium method $^{5}$ : After a sample was kept at $T_{\mathrm{s}}$ for $10 \mathrm{~min}$ in a solvent to attain diffusional equilibrium, it was cooled to $T_{\mathrm{b}}$ in the solvent at $5 \mathrm{~K} \mathrm{~min}^{-1}$, and then were kept at $T_{\mathrm{b}}$ for $10 \mathrm{~min}$. The temperature dependence of $\Delta n$ was measured at $5 \mathrm{~K} \mathrm{~min}^{-1}$. All samples and solvents, and the $T_{\mathrm{s}}$ and $T_{\mathrm{f}}$ are summarized in Table II.

Dynamic Viscoelasticities in a Solvent. Storage modulus $E^{\prime}$ and loss tangent $\tan \delta$ were measured with Rheovibron DDV-IIc (Toyo-Boldwin Co., Ltd., Japan) by the previous method $^{2}$ at $110 \mathrm{~Hz}$, and heating rate of $3 \mathrm{~K} \min ^{-1}$.

\section{RESULTS AND DISCUSSION}

\section{General Views of $\Delta n$ vs. Temperature Curve}

Figure 2 shows the temperature dependence of $\Delta n$ of BMM40(1) in methanol by the freezing and the equilibrium methods. $T_{\mathrm{s}}$ was fixed at $296 \mathrm{~K}$. We observe a few peaks in the case of the freezing method. In the freezing method, it should be noted that, $\Delta n$ was measured under the condition that solvent molecules cannot diffuse into the fine space among primary particles at the start and then diffusion proceeds with increasing temperature. In the case of the equilibrium method there were no obvious peaks until near $T_{\mathrm{s}}$, although there was 
one peak bellow $T_{\mathrm{s}}$. There has been no report on peaks of $\Delta n$ lower than $T_{\mathrm{s}}$. The appearance of peaks above $T_{\mathrm{s}}$ was related to the measuring method and solvent, and the peak location corresponded to the mechanical absorptions of $\beta_{\mathrm{a}}{ }^{3} \beta_{\mathrm{a}}$ absorption was due to local twisting motion of the main chain.

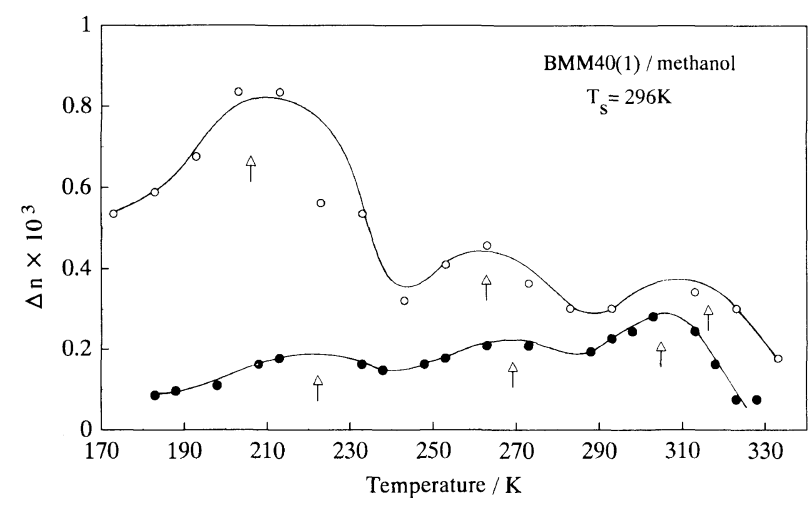

Figure 2. Temperature dependence of $\Delta n$ of BMM40(1) in methanol at $T_{\mathrm{s}}=296 \mathrm{~K}$ : Open circle, by the freezing method; filled circle, by the equilibrium method. Arrows indicate $\Delta n$ peak positions.

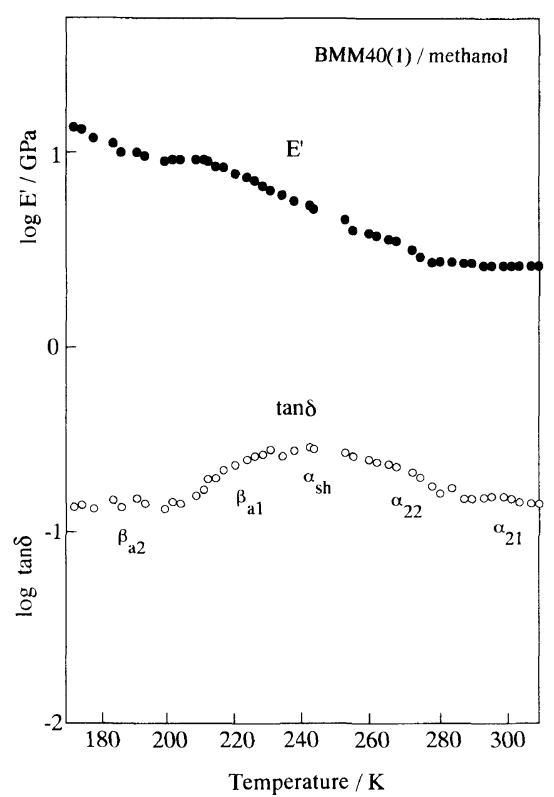

Figure 3. Temperature dependence of $E^{\prime}$ and $\tan \delta$ for BMM40(1) in methanol: Absorptions of $\beta_{\mathrm{a} 2}, \beta_{\mathrm{a} 1}, \alpha_{\mathrm{sh}}, \alpha_{22}$, and $\alpha_{21}$ locate at the temperature indicated in the figure.
Relationship between Thermal Molecular Motion and Appearance of a Peak in $\Delta n$ vs. Temperature Curve

Figure 3 shows the temperature dependence of dynamic viscoelasticity for BMM40(1) in methanol. The conditions of the measurement correspond to the equilibrium method in measurement of $\Delta n$. Five absorption peaks are observed in the curve of $\tan \delta v s$. temperature between melting and boiling temperatures of methanol: $\beta_{\mathrm{a} 2}, \beta_{\mathrm{a} 1}, \alpha_{\mathrm{sh}}, \alpha_{22}$, and $\alpha_{21}$ absorptions ${ }^{9}$ from lower temperature side, whose peaks were 193-213, $228-237,236-243,268$, and $288 \mathrm{~K}$, respectively. The mechanism of absorption is summarized in Table III based on the published literatures. ${ }^{2,9} \beta_{\mathrm{a}}$ absorption was used as a general term of $\beta_{\mathrm{a} 1}$ and $\beta_{\mathrm{a} 2}$ absorptions. By application of the theoretical equation to give peak temperature of the dynamic viscoelasticities, ${ }^{3}$ methanol molecules can diffuse into the region which gives the $\alpha_{2}$ absorption.

Figures 4(1) and 4(2) show the temperature dependence of $\Delta n$ of BMM40(1) and BMM40(2), respectively. Methanol was used as the immersing medium. Temperature between 170 and $240 \mathrm{~K}$ correspond to the range of $\beta_{\mathrm{a}}$ and $\alpha_{\mathrm{sh}}$ absorption in methanol. There can be observed three or more peaks in the case of the freezing method. The corresponding peaks are not observed in the case of the equilibrium one. The peak temperatures were located at $\beta_{\mathrm{a} 2}, \beta_{\mathrm{a} 1}$, and $\alpha_{\mathrm{sh}}$ absorption. We can conclude that mechanical absorption of $\beta_{\mathrm{a} 2}, \beta_{\mathrm{a} 1}$, and $\alpha_{\mathrm{sh}}$ gives the peak in $\Delta n v s$. temperature curve obtained by the freezing method by the use of methanol.

A comparison with BMM40(1) of Figure 4(1) and BMM40(2) of Figure 4(2), shows differences in the number of peaks of $\Delta n$ and levels of $\Delta n$. It is probable that structural change such as crystallinity changes with storage time cause the differences. The number of peaks depends on the number of distinguishable mechanical absorptions, such as the separation from $\beta_{\mathrm{a}}$ absorption into $\beta_{\mathrm{a} 2}$ and $\beta_{\mathrm{a} 1}$, and separation between $\beta_{\mathrm{a} 1}$ and $\alpha_{\mathrm{sh}}$. In this way, the peak number of $\Delta n$ for BMM40 is sensitively reflected to the fine structural change during storage. ${ }^{10}$

Figures 5(1) and 5(2) show the temperature dependence of $\Delta n$ for BMM40(1) and BMM40(2) between 240-340 $K$, respectively. This range corresponds to the range of $\alpha_{\mathrm{sh}}, \alpha_{22}$, and $\alpha_{21}$ absorptions in methanol. There can be observed a few peaks of $\Delta n$ in the case of freezing and equilibrium. $\Delta n$ peaks are closely related to $\alpha_{\mathrm{a}}$ adsorption.

Table III. Mechanism of mechanical absorption of a regenerated cellulose solid

\begin{tabular}{|c|c|c|c|c|c|}
\hline & sorptions & $T_{\max } / \mathrm{K}^{\mathrm{a}}$ & $\Delta H_{\mathrm{a}} / \mathrm{kJ} \mathrm{mol}^{-1 \mathrm{~b}}$ & Mechanism & Region $^{c}$ \\
\hline \multirow{4}{*}{$\alpha_{a}$} & $\alpha_{1}$ & $558-578$ & 1200 & Micro-Brownian motion of main chain & Intra-primary particle \\
\hline & $\alpha_{21}$ & $488-563$ & 290 & Ditto & Ditto \\
\hline & $\alpha_{22}$ & $413-563$ & 210 & Ditto & Ditto \\
\hline & $\alpha_{\mathrm{sh}}$ & $413-513$ & $C^{d}$ & Ditto & Interface of a primary particle \\
\hline & $\alpha_{\mathrm{H}_{2} \mathrm{O}}$ & $303-323$ & $-^{d}$ & $\begin{array}{l}\text { Co-operative motion of } \mathrm{H}_{2} \mathrm{O} \text { and } \\
\text { main chain }\end{array}$ & Intra-primary particle \\
\hline & $\mathrm{m}$ & 273 & $\infty$ & Melting of water adsorbed & Space among primary particles \\
\hline \multirow{2}{*}{$\beta_{\mathrm{a}}$} & $\beta_{\mathrm{a} 1}$ & 225 & 170 & Local twisting motion of main chain & Interface of a primary particle \\
\hline & $\beta_{\mathrm{a} 2}$ & 210 & 280 & Ditto & Intra-primary particle \\
\hline
\end{tabular}

${ }^{a}$ Peak temperature of $\tan \delta$ under dry air at $110 \mathrm{~Hz}$. ${ }^{\mathrm{b}}$ Apparent activation energy. ${ }^{\mathrm{c}}$ Region in which the polymer chains gives absorption. ${ }^{\mathrm{d}}$ Not decided. 


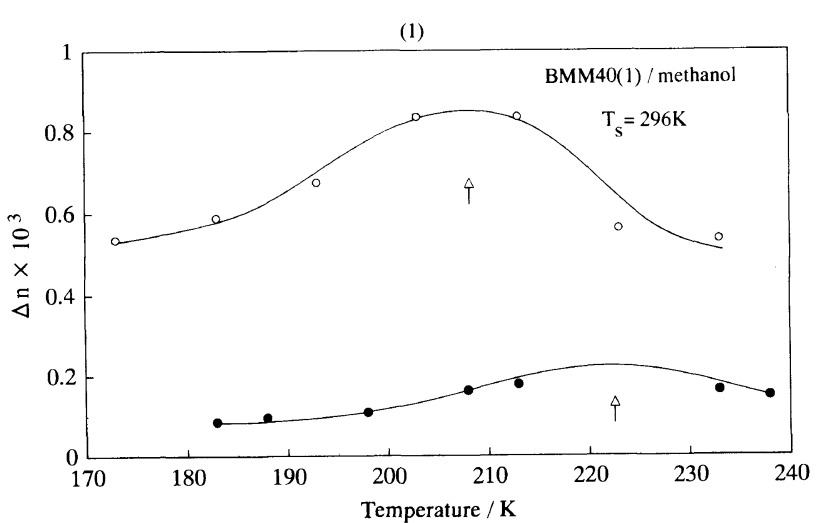

(2)

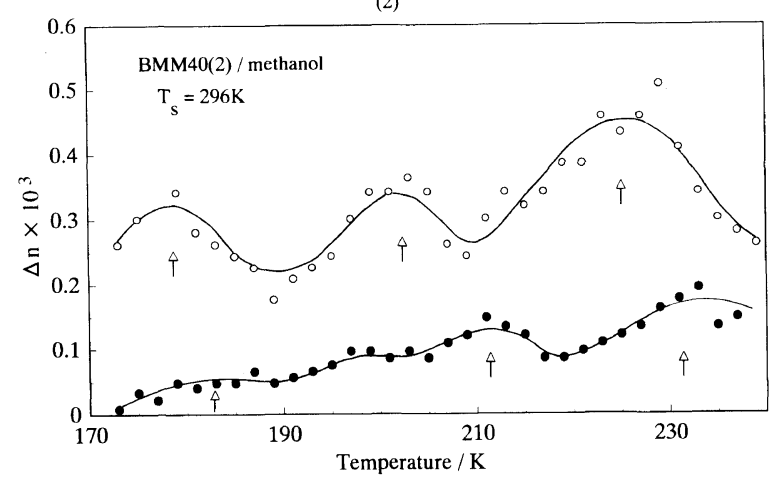

Figure 4. Tempeature dependence of $\Delta n$ of BMM40 in methanol at $T_{\mathrm{s}}=296 \mathrm{~K}$ : Open circle, by the freezing method; filled circle, by the equilibrium method. (1) BMM40(1); (2) BMM40(2). BMM40(2) indicates sample obtained by storing BMM40(1) in air of $\mathrm{RH}$ of $0 \%$ at $25^{\circ} \mathrm{C}$ for six months under free of tension. Arrows indicate $\Delta n$ peak positions.

\section{Occurrence of $\Delta n$ Peak}

$\Delta n$ Peak in the Range of $\beta_{\mathrm{a}}$ Absorption. In the case of the equilibrium method, no $\Delta n$ peak was observed or only small peaks were observed in the temperature range of $\beta_{\mathrm{a}}$ absorption. The temperature dependence of $\Delta n$, $\mathrm{d} \Delta n / \mathrm{d} T$ ( $T$; temperature) evaluated by this method in methanol for BMM40 and 35 was $6.3 \times 10^{-7}$ and $4.6 \times 10^{-6} \mathrm{~K}^{-1}$, respectively. $\beta_{\mathrm{a}}$ absorption gives this increase in $\Delta n$.

The $\Delta n$ peak in the case of the freezing method was always larger than that of the equilibrium method. The appearance of $\Delta n$ peak is considered to be related to non-equilibrium of the absorption of the solvent.

Judging from the principle of the freezing method, ${ }^{5}$ it is reasonable to assume that the above thermal molecular motion of cellulose chains is frozen at $T_{\mathrm{f}}$ of $173 \mathrm{~K}$. Methanol molecules do not yet diffuse into the inside of primary particles at $T_{\mathrm{f}}$, although they diffuse into voids among secondary particles. This diffusion was confirmed through the increase in the transparency of the sample. With increasing temperature, the thermal molecular motion of $\beta_{\mathrm{a}}$ absorption is released. Activated thermal motion may give rise to the diffusion of methanol molecules into the interface and then inside the primary particles. The diffusion of methanol on to the surface of the primary particle may give additional increase in $\Delta n$ since $\Delta n$ peak was larger than the case of the equilibrium method. This additional increase will be given in the forth-coming paper. ${ }^{12}$

In the case of the equilibrium method, the additional
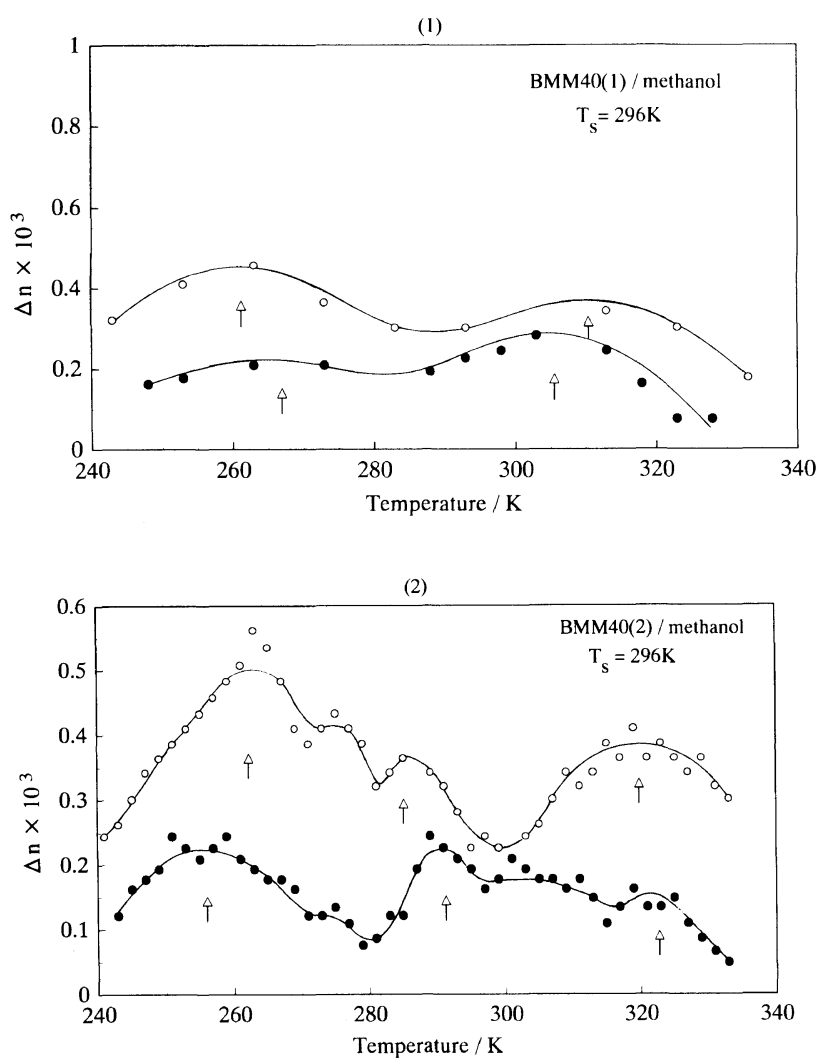

Figure 5. Temperature dependence of $\Delta n$ of BMM 40 in methanol at $T_{\mathrm{s}}=296 \mathrm{~K}$ : Open circle, by the freezing method; filled circle, by the equilibrium method. (1) BMM40(1); (2) BMM40(2). Arrows indicate $\Delta n$ peak positions.

diffusion of methanol into the cellulose solid may not occur at lower temperature than $T_{\mathrm{s}}(=296 \mathrm{~K})$. Because of completion of the diffusion into the interface of the primary particle, abrupt increase in $\Delta n$ with increasing temperature cannot be expected. Consequently, there are not observed any obvious peaks but a monotonous increase in $\Delta n$.

The appearance of peaks of $\Delta n$ obtained through the freezing method may be due to two opposite effects on $\Delta n$ : increase in $\Delta n$ with temperature mentioned above and the opposite. $\Delta n$ decreases toward values obtained by the equilibrium method at above temperature of $\beta_{\mathrm{a}}$ because of the concentration equilibrium of a methanol.

$\Delta n$ Peak in the Range of $\alpha_{\mathrm{a}}$ Absorption. The peak temperature and value changed with $T_{\mathrm{s}}$ in the equilibrium method and $T_{\mathrm{f}}$ in the freezing method. $\Delta n$ of the sample, obtained by cooling down to the room temperature after elevating above the peak temperature, did not coincide with the value before elevation. This indicates the $\Delta n$ peak is strongly related to irreversible change in the higher order structure.

Above $T_{\mathrm{s}}$, structural change due to the thermal molecular motion located at higher temperature than $T_{\mathrm{s}}$ may occur with increasing temperature. The appearance of peaks of $\Delta n$ may be due to the two opposite effects of positive temperature dependence of $\Delta n$ and a negative dependence. The positive dependence is caused by anisotropy of thermal expansion and anisotropic diffusion of a methanol molecule along a polymer chain axis. The negative dependence is caused by irreversible decrease in the chain orientation due to the segmental micro-Brownian motion. The micro-Brownian motion, 


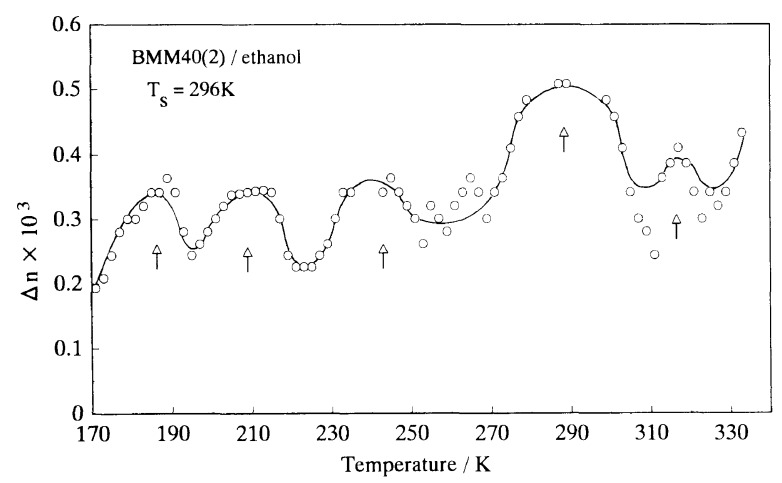

Figure 6. Temperature dependence of $\Delta n$ of BMM40(2) in ethanol by the freezing method: The arrows indicate the $\Delta n$ peak position.

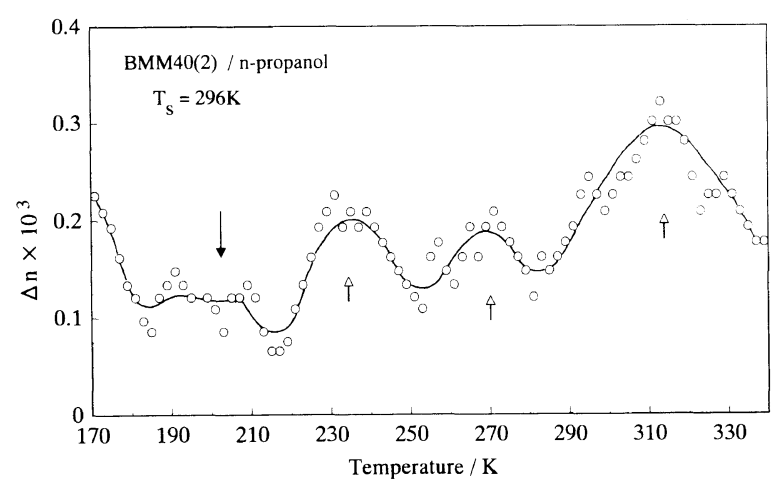

Figure 7. Temperature dependence of $\Delta n$ of BMM40(2) in $n$-propanol by the freezing method: Arrows with open mark indicate $\Delta n$ peak positions and one with closed mark indicates the $\Delta n$ minimum position.

Table IV. Peak temperatures of $\tan \delta\left(T_{\max }\right)$ in various solvents for BMM40

\begin{tabular}{|c|c|c|c|c|c|}
\hline \multirow[t]{2}{*}{ Solvent } & \multicolumn{5}{|c|}{$T_{\max } / \mathrm{K}$} \\
\hline & $\beta_{\mathrm{a} 2}$ & $\beta_{\mathrm{a} 1}$ & $\alpha_{\mathrm{sh}}$ & $\alpha_{22}$ & $\alpha_{21}$ \\
\hline Dry air & 200 & 230 & $378-473$ & $413-513$ & $488-563$ \\
\hline Methanol & $193-213$ & $228-237$ & $236-243$ & 268 & 288 \\
\hline Ethanol & ca. 210 & ca. 260 & ca. 290 & ca. 340 & \\
\hline n-Propanol & & $23^{\mathrm{a}}$ & $268-306$ & $338-343$ & 355 \\
\hline Ethylene glycol & & & $<300$ & 308 & 350 \\
\hline
\end{tabular}

${ }^{a}$ Not separated into two absorptions of $\beta_{\mathrm{a} 1}$ and $\beta_{\mathrm{a} 2}$.

activated by diffusion of a methanol molecule, gives rise to acceleration of the diffusion of methanol. A few peaks due to the combination of the positive and the negative dependence of $\Delta n$ were thus seen.

\section{Applications to Other Solvents}

Figure 6 shows the temperature dependence of $\Delta n$ of BMM40(2) in ethanol by the freezing method between 170 and $340 \mathrm{~K}$. There can be observed a few peaks in this temperature range. Table IV summarizes peak temperature $T_{\max }$ of $\tan \delta$ for BMM40 in several solvents. A broad and gentle peak of $\beta_{\mathrm{a}}$ absorption was not separated into two peaks, $\beta_{\mathrm{a} 1}$ and $\beta_{\mathrm{a} 2}$. For the curve of $\Delta n$ vs. temperature by the freezing method, the peaks corresponding to $\beta_{\mathrm{a}}$ adsorption are observed separately. The peaks of $\Delta n$ may always be expected whenever the onset of the thermal molecular motion with increasing temperature is realized.

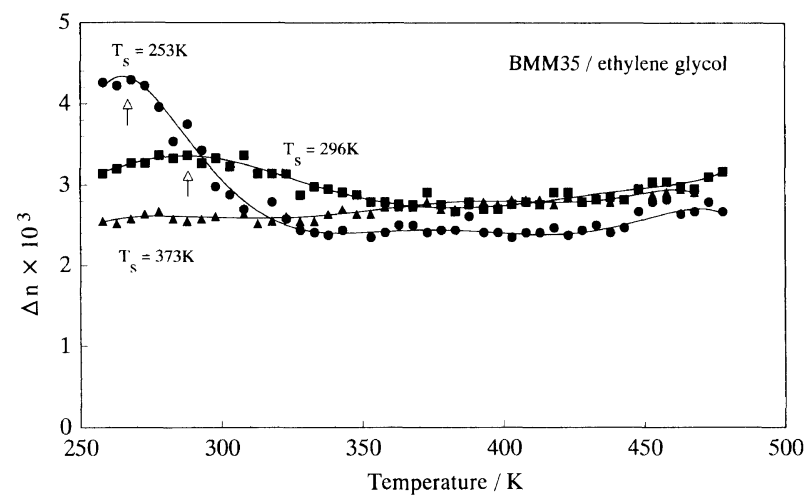

Figure 8. Temperature dependence of $\Delta n$ of BMM35 in ethylene glycol by the freezing method:,$T_{\mathrm{s}}=253 \mathrm{~K} ; \boldsymbol{\square}, 296 \mathrm{~K} ; \boldsymbol{\Delta}, 373 \mathrm{~K}$. Arrows indicate $\Delta n$ peak positions.

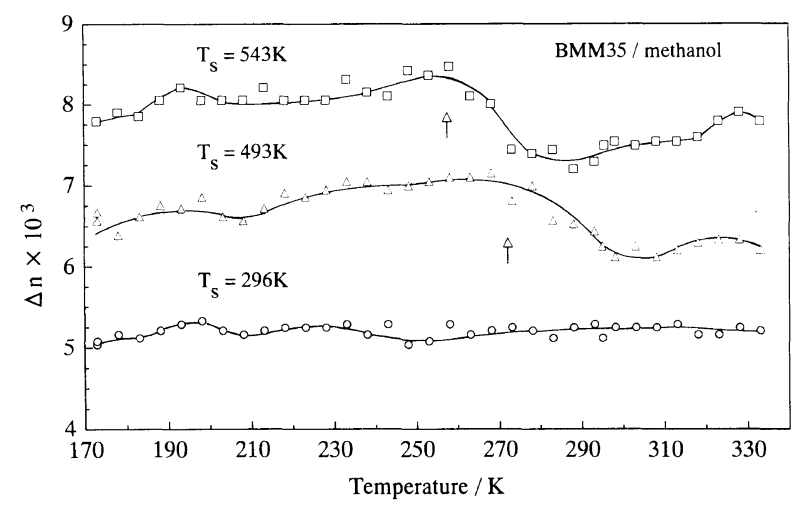

Figure 9. Temperature dependence of $\Delta n$ for BMM35 in methanol at $T_{\mathrm{s}}=543,493$, and $296 \mathrm{~K}$ by the freezing method: $\square, T_{\mathrm{s}}=543 \mathrm{~K} ; \triangle$, $493 \mathrm{~K} ; 0,296 \mathrm{~K}$. Arrows indicate $\Delta n$ peak positions.

Figure 7 shows the temperature dependence of $\Delta n$ of BMM 40(2) in $n$-propanol by the freezing method between 170 and $340 \mathrm{~K}$. A few peaks are observed. It is difficult to separate $\beta_{\mathrm{a}}$ absorption into two peaks, $\beta_{\mathrm{a} 1}$ and $\beta_{\mathrm{a} 2}$, in the case of $\tan \delta$ as shown in Table IV. By referring to Figures 4, 5, 6, and 7, we can conclude the value of $\Delta n$ peaks and their temperature location by the freezing method varied with the solvent.

Figure 8 shows the temperature dependence of $\Delta n$ of BMM35 in ethylene glycol by the equilibrium method. $T_{\mathrm{s}}$ are 253,296 , and $373 \mathrm{~K}$. The peak, located near $250 \mathrm{~K}$ in the case of $T_{\mathrm{s}}=253 \mathrm{~K}$, shifts to higher temperature side and the peak value falls with increasing $T_{s}$. The temperature range of this peak corresponds to the range between $\alpha_{22}$ and $\alpha_{21}$ absorptions in ethylene glycol. In the case of $T_{\mathrm{s}}$ of $373 \mathrm{~K}$, no peaks were observed. $\Delta n$ above the peak temperature increased with $T_{\mathrm{s}}$. when BMM35 attains diffusional equilibrium in ethylene glycol above $250 \mathrm{~K}$, the chain orientation is relaxed by activated thermal molecular motion resulting in decrease in $\Delta n$ near $250 \mathrm{~K}$. Change in $\Delta n$ above $T_{\mathrm{s}}$ is due to relaxation of the remaining chain orientation. The peak of $\Delta n$ is observed on the temperature of a few degrees higher than $T_{\mathrm{s}}$. The thermal molecular motion related to $\alpha_{22} \mathrm{ab}-$ sorption was completely activated in ethylene glycol at $T_{\mathrm{s}}=373 \mathrm{~K}$. Thus decrease in $\Delta n$ dose not take place in the temperature range of $\alpha_{22}$ absorption. From the experimental fact that $\Delta n$ after the peak increase with increasing $T_{\mathrm{s}}$, we can conclude that the heat treatment 
above $T_{\mathrm{s}}$ in ethylene glycol makes the chain orientation high.

Figure 9 shows the temperature dependence of $\Delta n$ for BMM 35 in methanol at $T_{\mathrm{s}}=543,493$, and $296 \mathrm{~K}$ by the freezing method. With increasing $T_{\mathrm{s}}, \Delta n$ below $\alpha_{\mathrm{a}}$ absorption increased greatly, and $\Delta n$ value above $\alpha_{\mathrm{a}}$ absorption increased slightly. The $\Delta n$ peak located near $250 \mathrm{~K}$ becomes greater and clear with an increase in $T_{\mathrm{s}}$. This temperature range of the peak covers the temperature range of $\alpha_{\mathrm{sh}}$ and $\alpha_{22}$ absorption. Thus, we can evaluate quantitatively changes in the higher order structure, such as the plane orientation originated by the solvent in freezing, and additional increase in $\Delta n$ due to the diffusion of solvents as showing quantitative analysis of the $\Delta n v s$. temperature curve.

Acknowledgments. We thank Mr. Y. Yano and Dr. K. Okajima in Asahi Chemical Ind. Co., Ltd. for financial support and encouragement.

\section{REFERENCES}

1. M. Mithuishi, Y. Naruoka, M. Shimizu, K. Hamada, and T Ishiwatari, J.S.D.C., 112, 333 (1996).

2. S. Manabe, M. Iwata, K. Kamide, Polym. J., 18, 1 (1986).

3. S. Manabe and R. Fujioka, Polym. J., 30, 939 (1998).

4. R. Fujioka and S. Manabe, Bull. Fac. Human Environ. Sci., Fukuoka Women's Univ., 27, 13 (1996).

5. R. Fujioka, Y. Inamasu, K. Umeda, and S. Manabe, Bull. Fac. Human Environ. Sci., Fukuoka Women's Univ., 28, 37 (1997).

6. for example R. S. Stein and F. H. Norris, J. Polym. Sci., 21, 381 (1956).

7. F. A. Bettelheim and R. S. Stein, J. Polym. Sci., 27, 567 (1958).

8. R. Fujioka, S. Manabe, and K. Imada, Bull. Fac. Eng., Miyazaki Univ., 26, 13 (1997)

9. S. Manabe and R. Fujioka, Polym. J., 28, 860 (1996).

10. R. Fujioka and S. Manabe, Sen'i Gakkaishi, 52, 516 (1996).

11. R. Fujioka, S. Manabe, and K. Imada, Bull. Fac. Eng., Miyazaki Univ., 27, 63 (1998).

12. R. Fujioka, A. Mori, S. Manabe, and K. Imada, Polym. J., to be submitted. 